\title{
ROJ
}

\section{Stereotactic radiotherapy of the prostate: fractionation and utilization in the United States}

\author{
Joseph P. Weiner, MD ${ }^{1,2}$, David Schwartz, MD ${ }^{1,3}$, Meng Shao, MD ${ }^{1,3}$, Virginia Osborn, MD ${ }^{1,3}$, \\ Kwang Choi, $\mathrm{MD}^{3}$, David Schreiber, MD ${ }^{1,3}$ \\ 'Department of Radiation Oncology, Veterans Affairs New York Harbor Healthcare System, Brooklyn, NY; \\ ${ }^{2}$ Department of Radiation Oncology, Stanford Cancer Institute, Stanford, CA; \\ ${ }^{3}$ Department of Radiation Oncology, SUNY Downstate Medical Center, Brooklyn, NY, USA
}

\begin{abstract}
Purpose: To analyze the utilization and fractionation of extreme hypofractionation via stereotactic body radiotherapy (SBRT) in the treatment of prostate cancer.

Materials and Methods: Data was analyzed on men diagnosed with localized prostate cancer between 2004-2012 and treated with definitive-intent radiation therapy, as captured in the National Cancer Database. This database is a hospital-based registry that collects an estimated 70\% of all diagnosed malignancies in the United States.

Results: There were 299,186 patients identified, of which 4,962 (1.7\%) were identified as receiving SBRT as primary treatment. Of those men, 2,082 had low risk disease (42.0\%), 2,201 had intermediate risk disease (44.4\%), and 679 had high risk disease (13.7\%). The relative utilization of SBRT increased from $0.1 \%$ in 2004 to $4.0 \%$ in 2012. Initially SBRT was more commonly used in academic programs, though as time progressed there was a shift to favor an increased absolute number of men treated in the community setting. Delivery of five separate treatments was the most commonly utilized fractionation pattern, with 4,635 patients (91.3\%) receiving this number of treatments. The most common dosing pattern was $725 \mathrm{cGy} \times 5$ fractions (49.6\%) followed by $700 \mathrm{cGy} \times 5$ fractions (21.3\%).

Conclusions: Extreme hypofractionation via SBRT is slowly increasing acceptance. Currently 700-725 cGy $\times 5$ fractions appears to be the most commonly employed scheme. As further long-term data regarding the safety and efficacy emerges, the relative utilization of this modality is expected to continue to increase.
\end{abstract}

Keywords: Prostate cancer, Stereotactic body radiation therapy, Fractionation, Utilization, National Cancer Database

\section{Introduction}

In the United States in 2015, an estimated 220,800 men were diagnosed with prostate cancer [1]. External beam radiation therapy (EBRT) has played an essential role in curative intent treatment of prostate cancer for the last half century [2], and contemporary data suggests that $24 \%-48 \%$ of newly diagnosed men will receive treatment with definitive radiation therapy [3]. Over the past 20 years, increasingly sophisticated delivery techniques, such as three-dimensional conformal radiation therapy (3DCRT) and intensity-modulated radiation therapy (IMRT), have allowed for the escalation of dose to

Received 14 November 2016, Revised 25 January 2017, Accepted 06 February 2017.

Correspondence: Joseph P. Weiner, MD, Department of Radiation Oncology, Veterans Administration New York Harbor Healthcare System, 800 Poly Place, Brooklyn, NY 11209, USA. Tel: +1-718-630-3605, Fax: +1-718-567-4082, E-mail: joseph. p.weiner@gmail.com

(c) This is an Open Access article distributed under the terms of the Creative Commons Attribution Non-Commercial License (http://creativecommons.org/ licenses/by-nc/4.0/) which permits unrestricted non-commercial use, distribution, and reproduction in any medium, provided the original work is properly cited.

www.e-roj.org 
the prostate with acceptable toxicity $[4,5]$. Since this time, several phase III trials have reported improved biochemical free survival with doses $>70 \mathrm{~Gy}$ [6], leading to the currently accepted standard dose of 75.6-81.0 cGy for conventionally fractionated radiation [7].

Though successful, conventionally-fractionated doseescalated treatment does have drawbacks such as prolonged treatment duration (8-9 weeks) as well as known toxicity to the bladder, bowel and erectile function [8]. In contrast to most other neoplasms, prostate cancer is thought to have a different intrinsic radiosensitivity to large doses per fraction as acknowledged via its low alpha/beta $(\alpha / \beta)$ ratio [9]. It is due to this that hypofractionation, the delivery of total radiation dose in larger fractions over a shorter period of time compared to conventionally fractionated radiation, is theoretically advantageous in producing tumor cell kill while reducing late toxicity in health cells [10]. This has been confirmed in practice by reports of several large retrospective analyses showing excellent disease free survival and low genitourinary and gastrointestinal toxicity [11-13]. In addition, the use of hypofractionation compared to brachytherapy may be more appropriate for patients that may be excluded from brachytherapy due to technical reasons such as pubic arch interference or prior transurethral resection of the prostate, and avoids the associated risks of anesthesia.

Therefore, due to the potential radiobiological and technical benefits, as well as being driven by patient convenience, hypofractionation is an attractive route for definitive treatment of the prostate. Accordingly, hypofractionation has been divided into two broad categories based on daily dose, either moderate (2.4-4 Gy) or extreme ( $\geq 6.5$ ). Extreme hypofractionation via teletherapy is delivered exclusively thru stereotactic body radiotherapy (SBRT). As defined by the American Society for Radiation Oncology (ASTRO), SBRT refers to treatment utilizing a precise position within a 3D space and consisting of five or fewer treatments [14]. Treatment commonly consists of multiple beams which tend to be non-opposing and are often non-coplanar with the goal of reducing entrance dose and normal tissue irradiated [15]. The purpose of this study is to analyze the utilization and patterns of care of delivery of extreme hypofractionation via SBRT in the treatment of prostate cancer in the United States.

\section{Materials and Methods}

\section{National Cancer Database}

The National Cancer Database (NCDB) is a hospital-based registry that is the joint project of the American Cancer Society and the Commission on Cancer of the American College of Surgeons. It is estimated that $70 \%$ of all diagnosed malignancies in the United States are captured by facilities participating in this registry and are reported to the NCDB. The Commission on Cancer's NCDB and the hospitals participating in the NCDB are the source of the de-identified data used in this study. However they have not verified and are not responsible for the statistical validity or conclusions derived by the authors of this study. Exemption was obtained from the New York Harbor Veterans Affairs Committee for Research and Development prior to the initiation of this study.

\section{Patient selection}

Men with prostate cancer diagnosed between 2004 and 2012 who were treated with either external beam radiation, and/ or prostate brachytherapy were abstracted from the NCDB. In order to be included, men had to be clinically staged as T1c$3 \mathrm{bNx}-0 \mathrm{MO}$ adenocarcinoma of the prostate. Complete data was necessary regarding Gleason score, T-stage, as well as prostate specific antigen in order to be included. This resulted in a total of 299,186 men. Patients were then grouped into one of five categories: SBRT alone, EBRT alone, brachytherapy alone, EBRT plus brachytherapy, or SBRT utilized as a boost. The primary focus of our analysis was on those who were categorized as receiving stereotactic radiotherapy alone to the prostate.

\section{Data analysis}

Descriptive statistics were used to analyze stereotactic radiotherapy utilization over time, as well as by other factors such as facility type and National Comprehensive Cancer Network (NCCN) risk grouping. Multivariate logistic regression was used to assess for predictors of stereotactic radiotherapy usage. The variables analyzed in the multivariate model were chosen based on reasonable factors that may potentially affect treatment choice. This included age grouping $(<60$ years, 60-70 years, $>70$ years), year of diagnosis (2004 through 2012 in single year increments), race (white, black, other), distance from treatment center (divided into 4 quartiles), facility type (academic, non-academic), insurance type (none, private insurance, Medicaid, Medicare, other government, unknown) and income level (divided into quartiles based on Census data). Significant values were defined as those with a $p$-value $<0.05$. Statistical analysis was performed using SPSS ver. 23 (IBM Inc., Armonk, NY, USA). 


\section{Results}

\section{Patient characteristics and SBRT prescription}

From the 299,186 men initially included in the study, 4,962 were identified as having received stereotactic radiotherapy alone. Of those, 2,082 (42.0\%) had NCCN low risk disease, 2,201 (44.4\%) had intermediate risk disease, and 679 (13.7\%) had high risk disease. The median age was 68 years (interquartile range, 63 to 73 years). Most men were white race and had Medicare or private insurance. The most common fractionation scheme included 5 fractions of radiation, with 725 cGy per fraction being the most frequently utilized fractionation scheme. Of those who received less than 5 fractions, the most common fractionation schedule was $950 \mathrm{cGy} \times 4$. The vast majority of men, 4,312 (86.9\%) were not treated with androgen deprivation therapy (ADT). Further details regarding patient characteristics are available in Table 1.

\section{Trends in prescription over time}

The relative utilization of stereotactic radiotherapy increased over time. SBRT alone and SBRT as a boost were both respectively utilized by $0.1 \%$ of all patients receiving radiation in 2004. This increased to 3.9\% for those receiving SBRT alone and $0.4 \%$ for those receiving SBRT as a boost by 2012. Fig. 1 summarizes these findings in addition to the usage of EBRT and brachytherapy as well, stratified yearly from 2004 to 2012.

\section{Factors affecting SBRT usage}

When analyzing SBRT utilization by NCCN risk grouping, there was an increase in use over time for all three risk groups, but a disproportionally larger absolute increase for low and intermediate versus high risk disease. For low risk disease, SBRT alone utilization increased from $0.1 \%$ in 2004 to 5.8\% in 2012. For intermediate risk disease, SBRT alone utilization increased from $0.1 \%$ to $4.3 \%$, and for high risk disease utilization increased from 0\% in 2004 to $1.7 \%$ in 2012.

Additionally, the proportion of men offered SBRT alone for the treatment of prostate cancer increased more quickly at academic institutions compared to non-academic institutions. In academic institutions, SBRT alone increased from 0.1\% in 2004 to $7.1 \%$ in 2012, whereas in non-academic facilities SBRT increased from $0.1 \%$ in 2004 to $2.4 \%$ in 2012. Although, absolute numbers of patients in the United States treated with SBRT initially favored academic institutions, but as time progressed a greater number of patients were treated outside of such facilities. In 2004, 22 men (81.5\%) and 5 men (18.5\%) were treated in academic versus non-academic facilities,
Table 1. Patient characteristics for those who received SBRT alone

\begin{tabular}{lc}
\hline \multicolumn{1}{c}{ Characteristic } & SBRT $(n=4,962)$ \\
\hline Age (yr) & \\
$<60$ & $751(15.1)$ \\
$60-70$ & $2,348(47.3)$ \\
$>70$ & $1,863(37.5)$ \\
Race & $4,161(83.9)$ \\
White & $657(13.2)$ \\
Black & $144(2.9)$ \\
Other & $2,082(42.0)$ \\
NCCN risk group & $2,201(44.4)$ \\
Low & $679(13.7)$ \\
Intermediate & \\
High & $2,311(46.6)$ \\
Facility type & $2,651(53.4)$ \\
Community & $1,681(33.9)$ \\
Academic & $54(1.1)$ \\
Insurance & $56(1.1)$ \\
Private & $3,024(60.9)$ \\
None & $58(1.2)$ \\
Medicaid & $89(1.8)$ \\
Medicare & \\
Other government & $4,312(86.9)$ \\
Unknown & $650(13.1)$ \\
Hormones & \\
No & $916(18.5)$ \\
Yes & $2,147(43.3)$ \\
Fractionation & $561(11.3)$ \\
700 cGy $\times 5$ & $341(6.9)$ \\
725 cGy $\times 5$ & $997(20.1)$ \\
750 cGy $\times 5$ & \\
$>750-1,000$ cGy $\times 5$ & \\
Other schemes ${ }^{2}$ & \\
Valesare &
\end{tabular}

Values are presented as number (\%).

SBRT, stereotactic body radiotherapy; NCCN, National Comprehensive Cancer Network.

${ }^{\text {a) }}$ Fewer than 5 fractions or missing data; the most common other fractionation scheme was $950 \mathrm{cGy} \times 4$ for 164 patients.

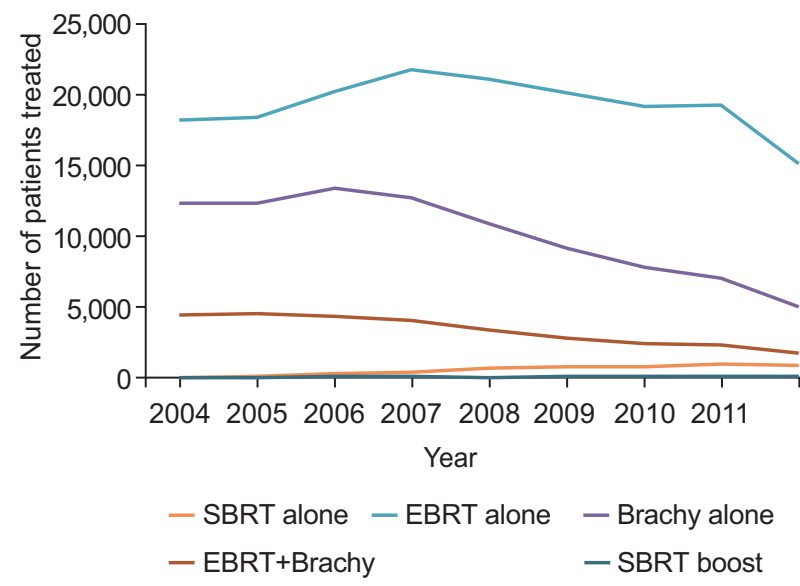

Fig. 1. Utilization of radiotherapy for treatment of prostate cancer over time. SBRT, stereotactic body radiotherapy; EBRT, external beam radiation therapy. 
Table 2. Utilization of SBRT based on NCCN risk group or treatment location

\begin{tabular}{|c|c|c|c|c|c|}
\hline \multirow{2}{*}{ Year } & \multicolumn{3}{|c|}{ Univariate } & \multicolumn{2}{|c|}{ Location of treatment } \\
\hline & Low & Intermediate & High & Academic & Community \\
\hline 2004 & $16(59.3)$ & $8(29.6)$ & $3(11.1)$ & $22(81.5)$ & $5(18.5)$ \\
\hline 2005 & $49(60.5)$ & $24(29.6)$ & 8 (9.9) & 61 (75.3) & $20(24.7)$ \\
\hline 2006 & $170(62.3)$ & $83(30.4)$ & $20(7.3)$ & $136(49.8)$ & $137(50.2)$ \\
\hline 2007 & $239(54.7)$ & $158(36.2)$ & $40(9.2)$ & $210(48.1)$ & $227(51.9)$ \\
\hline 2008 & $304(42.0)$ & $324(44.8)$ & $95(13.1)$ & $336(46.5)$ & $387(53.5)$ \\
\hline 2009 & $310(40.9)$ & $336(44.3)$ & $112(14.8)$ & 312 (41.2) & $446(58.8)$ \\
\hline 2010 & $319(38.4)$ & $385(46.4)$ & $126(15.2)$ & $373(44.9)$ & $457(55.1)$ \\
\hline 2011 & $359(37.8)$ & $434(45.7)$ & $157(16.5)$ & $479(50.4)$ & $471(49.6)$ \\
\hline 2012 & $316(35.8)$ & 449 (50.8) & $118(13.4)$ & $382(43.3)$ & $501(56.7)$ \\
\hline
\end{tabular}

Values are presented as number (\%).

NCCN, National Comprehensive Cancer Network.

though by 2012 it was noted that 382 men (43.3\%) and 501 men (56.7\%) were treated, respectively. SBRT use over time based on NCCN risk group and institution type is summarized on Table 2.

On multivariate logistic regression analysis, more recent year of diagnosis was the strongest predictor for SBRT usage. Compared to its usage in 2004, the odds ratio for SBRT increased to 2.47 (95\% confidence interval [Cl], 1.57-3.90; $p$ $<0.001)$ in 2005 and then further increased yearly through 2012, where the odds ratio was 59.52 (95\% Cl, 10.26-87.99; $p<0.001)$. Other factors were also associated with increased SBRT usage, though not as strongly as year of diagnosis. This included income level above the 50\% percentile, increased distance away from the treatment center, treatment at an academic facility, and Medicare insurance. Factors associated with a decreased likelihood of SBRT use included Medicaid or government insurance, and intermediate or high risk disease. Further details are available in Table 3.

\section{Discussion and Conclusion}

In our study, we utilized the NCDB to review the delivery of curative intent radiation therapy for prostate cancer via extreme hypofractionation in America men from 20042012. We were able to show that SBRT is slowly increasing acceptance over time. Academic facilities initially adapted this technique more readily, though in later years' community facilities were treating larger absolute numbers of patients. The majority of patients treated had either NCCN low or intermediate risk group disease and were not treated with androgen deprivation therapy. Currently, 700-725 cGy per fraction for 5 fractions appears to be the most commonly used scheme. Our findings are in agreement with other previously published reports on extreme hypofractionation in prostate cancer [16].

There are numerous hypothetical benefits to the application of SBRT for the treatment of prostate cancer. First, in the PSAera $>90 \%$ of all patients diagnosed with prostate cancer will present with localized disease [1]. This allows for a smaller treatment volume to be targeted, thus exploiting the inherent precision of SBRT-based treatment, and decreasing a patient's integral dose. Second, it is generally accepted by radiobiologists that the $\alpha / \beta$ ratio, which compares the intrinsic radiosensitivity of non-repairable cell kill versus repairable cell kill, is as low as $1.5 \mathrm{~Gy}$ in contrast to $3 \mathrm{~Gy}$ for late responding tissues and 10 Gy for most other tumors and acute responding tissues [17]. By having a lower $\alpha / \beta$ ratio, the treatment of prostate cancer with hypofractionation theoretically takes advantage of tumor kill with potential sparing of late tissue complications versus conventionally fractionated treatment [18].

However, despite such theoretical benefits two recently reported hypofractionation trials have had some mixed outcomes. The CHHiP trial was a randomized, phase 3 noninferiority trial performed in the United Kingdom comparing two moderately hypofractionated regimens (60 Gy in 20 fractions and 57 Gy in 19 fractions) versus a conventional 2 Gy per day regimen to 74 Gy [19]. The 60 Gy regimen was found to be non-inferior to the conventionally fractioned scheme, though the 57 Gy regimen was not. Patient-reported outcomes and toxicity were found to be similar 24 months after treatment [20]. The HYPRO trial was a randomized, phase 3 trial enrolling localized but intermediate to high risk patients randomly assigned to either a moderately hypofractionated (64.6 Gy in 19 fractions) or conventionally fractionated (78.0 Gy in 39 fractions) regime [21]. Five-year outcomes including relapse-free survival were not different, though an 
Table 3. Patient characteristics for those who received SBRT alone

\begin{tabular}{|c|c|c|}
\hline Characteristic & OR $(95 \% \mathrm{Cl})$ & $p$-value \\
\hline \multicolumn{3}{|l|}{ Age group (yr) } \\
\hline$<60$ & 1 & \\
\hline $60-70$ & $1.04(0.95-1.15)$ & 0.35 \\
\hline$>70$ & $1.02(0.92-1.14)$ & 0.68 \\
\hline \multicolumn{3}{|l|}{ Year of diagnosis } \\
\hline 2004 & 1 & \\
\hline 2005 & $2.47(1.57-3.90)$ & $<0.001^{*}$ \\
\hline 2006 & $9.11(6.08-13.65)$ & $<0.001^{*}$ \\
\hline 2007 & $14.43(9.70-21.46)$ & $<0.001^{*}$ \\
\hline 2008 & $28.18(19.04-41.70)$ & $<0.001^{*}$ \\
\hline 2009 & $32.99(22.30-48.81)$ & $<0.001^{*}$ \\
\hline 2010 & $40.35(27.29-59.67)$ & $<0.001^{*}$ \\
\hline 2011 & $47.72(32.30-70.51)$ & $<0.001^{*}$ \\
\hline 2012 & $59.48(40.24-87.94)$ & $<0.001^{*}$ \\
\hline \multicolumn{3}{|l|}{ Race } \\
\hline White & 1 & \\
\hline Black & $0.95(0.86-1.04)$ & 0.23 \\
\hline Other & $0.58(0.49-0.68)$ & $<0.001^{*}$ \\
\hline \multicolumn{3}{|c|}{ Distance from treatment center } \\
\hline First quartile (closest) & 1 & \\
\hline Second quartile & $1.04(0.95-1.14)$ & 0.38 \\
\hline Third quartile & $1.20(1.10-1.31)$ & $<0.001^{*}$ \\
\hline Last quartile (furthest) & $1.78(1.64-1.94)$ & $<0.001^{*}$ \\
\hline \multicolumn{3}{|l|}{ Facility type } \\
\hline Non-academic program & 1 & \\
\hline Academic program & $2.73(2.57-2.90)$ & $<0.001^{*}$ \\
\hline \multicolumn{3}{|l|}{ Insurance } \\
\hline Private insurance & 1 & \\
\hline None & $0.96(0.72-1.27)$ & 0.76 \\
\hline Medicaid & $0.66(0.51-0.87)$ & $0.003^{*}$ \\
\hline Medicare & $1.43(1.33-1.54)$ & $<0.001^{*}$ \\
\hline Other government & $0.51(0.39-0.67)$ & $<0.001^{*}$ \\
\hline Unknown & $1.39(1.11-1.73)$ & $0.004^{*}$ \\
\hline \multicolumn{3}{|l|}{ Income } \\
\hline First quartile (lowest) & 1 & \\
\hline Second quartile & $1.04(0.93-1.16)$ & 0.54 \\
\hline Third quartile & $1.30(1.17-1.44)$ & $<0.001^{*}$ \\
\hline Last quartile (highest) & $2.31(2.09-2.55)$ & $<0.001^{*}$ \\
\hline
\end{tabular}

$\mathrm{SBRT}$, stereotactic body radiotherapy; $\mathrm{OR}$, odds ratio; $\mathrm{Cl}$, confidence interval.

${ }^{*} \mathrm{p}<0.05$.

increased cumulative incidence of grade 2 or worse acute gastrointestinal toxicity was noted in the hypofractionation regimen [22]. As such, the authors conclude that their hypofractionated regimen was not superior to conventional fractionation.

Regardless, our study showed a consistent increase in the use of SBRT from 2004 to 2012, increasing from 0.1\% to $3.9 \%$ for all patients treated with radiation $(0.2 \%$ to $4.3 \%$ if including SBRT as a boost). The observed increased of SBRT utilization is likely multifactorial. Both the potential radiobiological benefits, as well as patient convenience, make hypofractionation an attractive option for definitive treatment to both caregivers and patients. Additionally, given increased attention to health care costs, the use of SBRT may be an especially attractive modality. A recent paper by Halpern et al. [23] used SEER-Medicare linked data to estimate the median cost of different treatment modalities for localized prostate cancer. Of all teletherapy modalities, SBRT was found to be the least expense at $\$ 27,145$ per course compared with $\$ 37,090$ for IMRT and $\$ 54,706$ for proton beam therapy.

Our results show a substantial variation in the probability of receiving SBRT among those who were irradiated. Certain factors were noted to favor an increased adoption of SBRT, including treatment at an academic center. Such centers tend to be early adopters of new technology, especially when adoption includes resource-heavy changes. Successful implementation of an SBRT program is highly dependent on physician training specialized physics support and potentially costly upgrades in machinery and staff.

Patient specific demographics were also a factor in decreased likelihood of receiving SBRT including those in lower income brackets and black race. This is consistent with prior research showing that lower-income and black cancer patients are less likely to receive oncologic treatment [24]. Interestingly, patients noted to live furthest from their treatment center were around 80\% more likely to have received SBRT compared to those that lived closest. The convenience of the treatment schedule compared to conventionally fractioned EBRT may be a factor in the decision process for such patients. Perhaps it will even effect decision making with respect to a patient's desire to opt for which type of definitive intent treatment or even treatment all together. A recent study by Muralidhar et al. [25] showed that patients living in urban or rural areas were less likely to receive radiation therapy compared to surgery if they lived father from a treatment facility. Hypofractionation may combat this disparity and allow for an increased likelihood of selecting radiotherapy for these patients.

As evident by this study, the past decade has shown a dramatic change in the landscape of the delivery of radiation for prostate cancer. Nevertheless, currently only between $0.1 \%-0.4 \%$ of patients in our study received SBRT as a boost in combination with an additional radiation modality. Future interest with SBRT may not be limited to its use as monotherapy due to extrapolation from emerging research in prostate brachytherapy. Recent studies have suggested improved outcomes with further dose escalation via combination brachytherapy plus conventionally fractionated EBRT versus EBRT alone, especially in intermediate and high risk patients [26,27]. As reported by Spratt et al. [28] in their study of EBRT alone (86.4 Gy) versus combination therapy 
(EBRT 45-50.4 Gy + 100-110 Gy brachytherapy boost), they calculated an EQD2 of 82 Gy for EBRT alone and $110 \mathrm{~Gy}$ for combination treatment and showed a greater than 10\% PSA relapse free survival at 7 years. Granted, one should be mindful of the question of applicability of the linear-quadratic model at large doses per fraction [29], as it has been postulated that different categories of cell kill besides mitotic death, such as endothelial cell death or tumor-associated antigen release with enhanced immune response [30], may also occur.

Finally, it is important to note that our study does have several limitations. First, the data used for analysis was registry-based from the NCDB, which therefore inherently limits which data is collected and is thus reportable in our results. For example, information such as the type of image guidance or fraction spacing could not be reliably reviewed. Furthermore, while the NCBD represents one of the most complete catalogues of information for contemporarily treated cancer patients, it only collects an estimated $70 \%$ of all US cancer patients. As such, nearly a third of all cancer encounters are not represented in our reported findings. In addition, although recent literature [23] also suggests an increased use of SBRT over time, we are unable to extend direct conclusions about SBRT utilization more recently than 2012, as this was the latest year for which data was available to be included in our analysis. Also this data has not yet matured, limiting our ability to report on long-term efficacy and toxicity of this treatment. In addition, this data is also associated with the inherit treatment and information biases always present in retrospective analyses. Additionally, the majority of patients in our study were not treated with ADT. Historically, ADT was largely excluded from the major dose escalation studies [31,32], so the role of dose escalation with ADT is less clear in even the conventionally fractionated setting. Thus the role of ADT with extreme hypofractionation is even less well established and will be an area of future research, particular for our highest risk patients.

In conclusion, extreme hypofractionation via SBRT is slowly increasing in utilization for the treatment of definitive prostate cancer. Current patterns of care suggest that 700-725 cGy per fraction for 5 fractions appears to be the most commonly utilized fractionation scheme in the United States. Though the relative utilization of SBRT is expected to continue to increase, questions about the long-term safety, efficacy and proper integration with multimodality treatment exists.

\section{Conflict of Interest}

No potential conflict of interest relevant to this article was reported.

\section{References}

1. Siegel RL, Miller KD, Jemal A. Cancer statistics, 2015. CA Cancer J Clin 2015;65:5-29.

2. Bagshaw MA, Kaplan HS, Sagerman RH. Linear accelerator supervoltage radiotherapy. VII. Carcinoma of the prostate. Radiology 1965;85:121-9.

3. DeSantis $C E$, Lin $C C$, Mariotto $A B$, et al. Cancer treatment and survivorship statistics, 2014. CA Cancer J Clin 2014;64:252-71.

4. Leibel SA, Zelefsky MJ, Kutcher GJ, et al. The biological basis and clinical application of three-dimensional conformal external beam radiation therapy in carcinoma of the prostate. Semin Oncol 1994:21:580-97.

5. Ling CC, Burman $\mathrm{C}$, Chui $\mathrm{CS}$, et al. Conformal radiation treatment of prostate cancer using inversely-planned intensity-modulated photon beams produced with dynamic multileaf collimation. Int J Radiat Oncol Biol Phys 1996;35:721-30

6. Zietman AL, DeSilvio ML, Slater JD, et al. Comparison of conventional-dose vs high-dose conformal radiation therapy in clinically localized adenocarcinoma of the prostate: a randomized controlled trial. JAMA 2005;294:1233-9.

7. National Comprehensive Cancer Network. NCCN Guidelines: prostate cancer version 3, 2016 [Internet]. Fort Washington, PA: National Comprehensive Cancer Network; c2017 [cited at 2017 May 15]. Available from: https://www.ncen.org/ professionals/physician_gls/f_guidelines.asp.

8. Xu N, Rossi PJ, Jani AB. Toxicity analysis of dose escalation from 75.6 Gy to 81.0 Gy in prostate cancer. Am J Clin Oncol 2011;34:11-5

9. Williams MV, Denekamp J, Fowler JF. A review of alphal beta ratios for experimental tumors: implications for clinical studies of altered fractionation. Int J Radiat Oncol Biol Phys 1985;11:87-96.

10. Nahum AE. The radiobiology of hypofractionation. Clin Oncol (R Coll Radiol) 2015;27:260-9.

11. Madsen BL, Hsi RA, Pham HT, Fowler JF, Esagui L, Corman J. Stereotactic hypofractionated accurate radiotherapy of the prostate (SHARP), 33.5 Gy in five fractions for localized disease: first clinical trial results. Int J Radiat Oncol Biol Phys 2007;67:1099-105.

12. King $C R$, Freeman D, Kaplan I, et al. Stereotactic body 
radiotherapy for localized prostate cancer: pooled analysis from a multi-institutional consortium of prospective phase II trials. Radiother Oncol 2013;109:217-21.

13. Bolzicco G, Favretto MS, Satariano N, Scremin E, Tambone $C$, Tasca A. A single-center study of 100 consecutive patients with localized prostate cancer treated with stereotactic body radiotherapy. BMC Urol 2013;13:49.

14. Buyyounouski MK, Price RA Jr, Harris EE, et al. Stereotactic body radiotherapy for primary management of early-stage, low- to intermediate-risk prostate cancer: report of the American Society for Therapeutic Radiology and Oncology Emerging Technology Committee. Int J Radiat Oncol Biol Phys 2010;76:1297-304.

15. Kavanagh BD, Timmerman RD, Benedict SH, et al. How should we describe the radiobiologic effect of extracranial stereotactic radiosurgery: equivalent uniform dose or tumor control probability? Med Phys 2003;30:321-4.

16. Lischalk JW, Kaplan ID, Collins SP. Stereotactic body radiation therapy for localized prostate cancer. Cancer J 2016;22:30713.

17. Fowler JF. The radiobiology of prostate cancer including new aspects of fractionated radiotherapy. Acta Oncol 2005;44:26576.

18. Duchesne GM, Peters LJ. What is the alpha/beta ratio for prostate cancer? Rationale for hypofractionated high-doserate brachytherapy. Int J Radiat Oncol Biol Phys 1999;44:7478.

19. Dearnaley D, Syndikus I, Mossop H, et al. Conventional versus hypofractionated high-dose intensity-modulated radiotherapy for prostate cancer: 5-year outcomes of the randomised, noninferiority, phase 3 CHHiP trial. Lancet Oncol 2016;17:104760.

20. Wilkins A, Mossop H, Syndikus I, et al. Hypofractionated radiotherapy versus conventionally fractionated radiotherapy for patients with intermediate-risk localised prostate cancer: 2-year patient-reported outcomes of the randomised, noninferiority, phase 3 CHHiP trial. Lancet Oncol 2015;16:160516.

21. Incrocci L, Wortel RC, Alemayehu WG, et al. Hypofractionated versus conventionally fractionated radiotherapy for patients with localised prostate cancer (HYPRO): final efficacy results from a randomised, multicentre, open-label, phase 3 trial.
Lancet Oncol 2016;17:1061-9.

22. Aluwini S, Pos F, Schimmel E, et al. Hypofractionated versus conventionally fractionated radiotherapy for patients with prostate cancer (HYPRO): late toxicity results from a randomised, non-inferiority, phase 3 trial. Lancet Oncol 2016;17:464-74.

23. Halpern JA, Sedrakyan A, Hsu WC, et al. Use, complications, and costs of stereotactic body radiotherapy for localized prostate cancer. Cancer 2016;122:2496-504.

24. Hardy D, Liu CC, Xia R, et al. Racial disparities and treatment trends in a large cohort of elderly black and white patients with nonsmall cell lung cancer. Cancer 2009;115:2199-211.

25. Muralidhar V, Rose BS, Chen YW, Nezolosky MD, Nguyen PL. Association between travel distance and choice of treatment for prostate cancer: does geography reduce patient choice? Int J Radiat Oncol Biol Phys 2016;96:313-7.

26. Xiang $M$, Nguyen PL. Significant association of brachytherapy boost with reduced prostate cancer-specific mortality in contemporary patients with localized, unfavorable-risk prostate cancer. Brachytherapy 2015;14:773-80.

27. Muralidhar $V$, Xiang M, Orio PF 3rd, et al. Brachytherapy boost and cancer-specific mortality in favorable high-risk versus other high-risk prostate cancer. J Contemp Brachytherapy 2016;8:1-6.

28. Spratt DE, Zumsteg ZS, Ghadjar P, et al. Comparison of highdose (86.4 Gy) IMRT vs combined brachytherapy plus IMRT for intermediate-risk prostate cancer. BJU Int 2014;114:360-7.

29. Brown JM, Carlson DJ, Brenner DJ. The tumor radiobiology of SRS and SBRT: are more than the 5 Rs involved? Int J Radiat Oncol Biol Phys 2014;88:254-62.

30. Song CW, Kim MS, Cho LC, Dusenbery K, Sperduto PW. Radiobiological basis of SBRT and SRS. Int J Clin Oncol 2014;19:570-8.

31. Zietman AL, Bae K, Slater JD, et al. Randomized trial comparing conventional-dose with high-dose conformal radiation therapy in early-stage adenocarcinoma of the prostate: longterm results from Proton Radiation Oncology Group/American College of Radiology 95-09. J Clin Oncol 2010;28:1106-11.

32. Kuban DA, Tucker SL, Dong $L$, et al. Long-term results of the M. D. Anderson randomized dose-escalation trial for prostate cancer. Int J Radiat Oncol Biol Phys 2008;70:67-74. 